SHORT REPORT

\title{
Contralateral pharyngeal paralysis caused by medial medullary infarction
}

\author{
M Nakajima, M Inove, Y Sakai
}

J Neurol Neurosurg Psychiatry 2005;76:1292-1293. doi: 10.1136/jnnp.2004.058586

A patient with unilateral upper medial medullary infarction presented with contralateral paralysis of the pharyngeal constrictor muscle in association with lemniscal sensory loss, pyramidal insufficiency, and central facial palsy on the same side. Individual differences in supranuclear control of the pharyngeal muscles may explain this rare occurrence. The combination of these signs is described as a syndrome of upper medial medullary lesion.

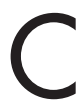
linical manifestations of medial medullary infarction (MMI) vary, unlike those of the classical Déjerine syndrome. ${ }^{1-4}$ Ipsilateral hypoglossal palsy does not occur often, and contralateral hemiparesis and lemniscal sensory loss do not always appear. In contrast, contralateral central facial palsy, not seen in the classic description, is found in a significant number of patients with MMI. ${ }^{5}$ This contralateral central facial palsy is in contrast to the ipsilateral type present in lateral medullary infarction (LMI). ${ }^{5}$ Dysphagia associated with palatal and pharyngeal weakness is rare in MMI, but has been reported in bilateral MMI and in the combination of MMI with LMI. ${ }^{4-9}$ We report a patient with unilateral MMI who presented with contralateral paralysis of the upper pharyngeal constrictor muscle in association with other MMI features.

\section{CASE REPORT}

A 53 year old right handed man experienced heaviness of the right hand, then minimal speech and deglutition difficulties of acute onset. By the next day, the heaviness involved both the entire right upper and lower limbs, and clumsiness of the right hand and unstable gait followed. He had diabetes mellitus, which had been controlled medically for 4 years. On examination, he was conscious and fully oriented. His pupils were equal and reacted to light. Eye movement was normal, and there was no nystagmus. Trigeminal sensory and motor functions were normal. There was minimal weakness of the

Abbreviations: LMI, lateral medullary infarction; MMI, medial medullary infarction; MRI, magnetic resonance imaging; TMS, transcranial magnetic stimulation; MEP, motor evoked potential
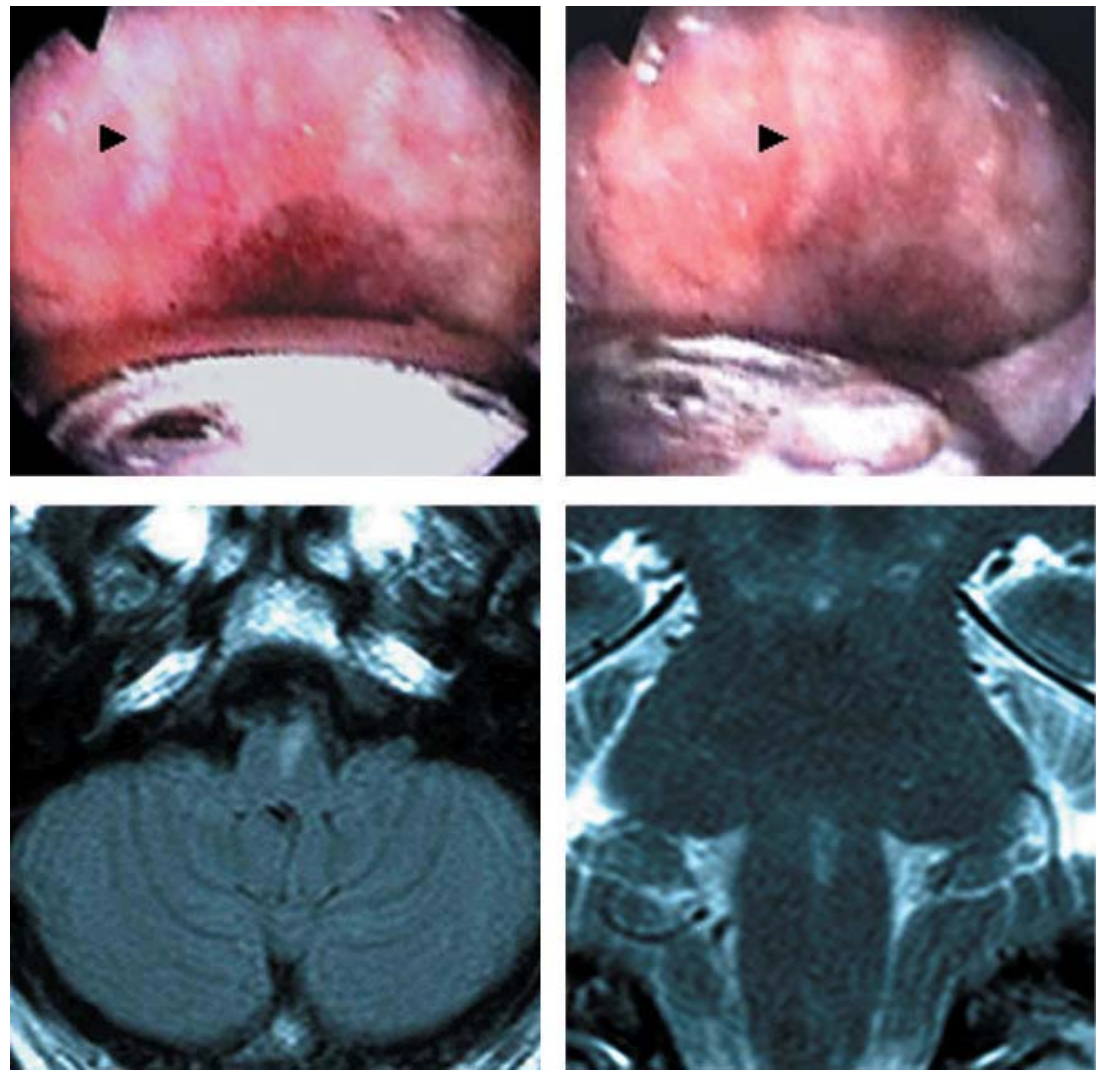

Figure 1 (A) Posterior wall of the pharynx seen through the oral cavity at rest (left) and while attempting to phonate (right). The posterior wall has shifted to the patient's left because of paralysis of the right blade of the upper pharyngeal constrictor muscle. The arrowhead in each view indicates the same mucosal ridge on the right side of the pharyngeal wall. (B) Magnetic resonance image of the fluid attenuated inversion recovery sequence for the axial (left) and T2 weighted coronal (right) sections. There is a well demarcated unilateral medial medullary infarct just below the pontomedullary junction. The right side of each image corresponds to the left side of the brain. 
lower half of the face on the right, as evidenced by impaired elevation of the upper lip when he opened his mouth. His head inclined slightly posterolaterally towards the left, but vestibular function otherwise was normal. While attempting to phonate, the posterior pharyngeal wall moved to the left, indicative of paralysis of the right side of the upper pharyngeal constrictor muscle (signe de rideau of Vernet ${ }^{10}$ ) (fig lA). The soft palate was normal in position and motion, but there was no soft palate reflex on either side. There was no tongue paralysis, and laryngeal examination showed normal vocal cord motion. In the limbs, muscle strength was normal and symmetrical. On the right side, muscle stretch reflexes were exaggerated, and there was extensor plantar response. Thermal and pinprick sensations in the limbs were unimpaired. Perception of joint movement and vibration was impaired in the right fingers and toes. Limb ataxia, present on the right, worsened when visual guidance was deprived. Magnetic resonance imaging of the brain showed a left side medial medullary infarction just below the pontomedullary junction (fig 1B). The rest of the brain stem and cerebellum, the hemispheres, and the cranial base otherwise were normal. Magnetic resonance angiography of the brain showed significant stenosis of the left vertebral artery. Routine laboratory tests and echocardiography were normal except for elevated blood sugar and haemoglobin $\mathrm{A}_{1 \mathrm{C}}$ levels. After a course of medical treatment, stable gait was restored. Minimal dysarthria and dysphagia lasted only a few days, whereas the right sided, central type facial and pharyngeal weakness still persisted at the follow up examination 2 months after stroke onset.

\section{DISCUSSION}

This patient had persistent paralysis of the right side of the upper posterior pharyngeal constrictor muscle due to a well demarcated, left sided medial medullary infarct. Unilateral motor weakness of the pharyngeal muscle, which is innervated by the nucleus ambiguus, usually indicates a nuclear or infranuclear lesion, but unilateral paralysis of the palatal, pharyngeal, and laryngeal muscles caused by a supranuclear lesion occasionally has been reported. Unilateral faciolingual-masseter-pharyngeal-laryngeal weakness from a contralateral opercular infarction was reported in the $1960 \mathrm{~s},{ }^{11}$ but no additional cases have been reported since brain imaging techniques have come into use. In 1990, Bogousslavsky and Regli ${ }^{12}$ reported six patients with facial and lingual hemiparesis due to a contralateral lesion limited to the genu of the internal capsule, originally described by Déjerine and Déjerine-Klumpke. ${ }^{13}$ On the same side as their faciolingual hemiparesis, three patients showed unilateral weakness of the masseter muscle associated with palatal weakness (two), pharyngeal hypotonia and vocal cord paralysis (one), and decreased pharyngeal reflex (one). A review of the literature turned up two previous reports of unilateral paralysis of muscle(s) innervated by the nucleus ambiguus contralateral to a lesion in the corona radiata, ${ }^{14}$ and in the genu of the internal capsule. ${ }^{15}$ In some individuals therefore, the nucleus ambiguus seems to be governed by crossed corticonuclear innervation. A transcranial magnetic stimulation study of humans by Ertekin et al suggested that in the nucleus ambiguus there is a crossed corticofugal connection. ${ }^{16}$ Motor evoked potentials were elicited in the cricothyroid muscle after contralateral motor cortex stimulation.

Our patient had unilateral, probably supranuclear, paralysis of the upper pharyngeal constrictor muscle, which has not been reported in medial medullary syndrome. The case of our patient further suggests that in humans supranuclear control of the nucleus ambiguus may have contalateral predominance.

\section{Authors' affiliations \\ M Nakajima, M Inove, Department of Neurology, Tokyo Rosai \\ Occupational Diseases and Injuries Hospital, Tokyo, Japan \\ Y Sakai, Department of Otorhinolaryngology, Tokyo Rosai \\ Occupational Diseases and Injuries Hospital \\ Competing interests: none declared}

Correspondence to: Dr M Nakajima, Department of Neurology, Tokyo Rosai Occupational Diseases and Injuries Hospital, 4-13-21 Ohmoriminami, Ohta-ku, Tokyo, Japan 143-0013; masashi@tokyoh.rofuku. go.jp

Received 10 November 2004

In revised form 17 January 2005

Accepted 17 January 2005

\section{REFERENCES}

1 Kim JS, Kim HG, Chung CS. Medial medullary syndrome. Report of 18 new patients and a review of the literature. Stroke 1995;26:1548-52.

2 Toyoda K, Imamura T, Saku Y, et al. Medial medullary infarction: analysis of eleven patients. Neurology 1996;47:1141-7.

3 Bassetti C, Bogousslavsky J, Mattle H, et al. Medial medullary stroke: report of seven patients and review of the literature. Neurology 1997;48:882-90.

4 Kumral E, Afsar N, Kirbas D, et al. Spectrum of medial medullary infarction: clinical and magnetic resonance imaging findings. J Neurol 2002;249:85-93

5 Urban PP, Wicht S, Vucorevic G, et al. The course of corticofacial projections in the human brainstem. Brain 2001;124:1866-76.

6 Meyer JS, Herndon RM. Bilateral infarction of the pyramidal tracts in man. Neurology 1962;12:637-42.

7 Currier RD. The medial medullary syndrome. J Univ Mich Med Cen 1976;42:96-104.

8 Mizutani T, Lewis RA, Gonatas NK. Medial medullary syndrome in a drug abuser. Arch Neurol 1980;37:385-7.

9 Tyler KL, Sandberg E, Baum KF. Medial medullary syndrome and meningovascular syphilis: a case report in an HIV-infected man and a review of the literature. Neurology 1994;44:2231-5.

10 Vernet M. Les paralysies laryngés associées. Lyon: Thèse, 1916:49-55.

11 Rondot P. Syndromes of central motor disorder. In: Vinken PJ, Bruyn GW, eds. Handbook of clinical neurology, vol 1. New York: Elsevier, 1969:169-217.

12 Bogousslavsky J, Regli F. Capsular genu syndrome. Neurology 1990;40: 1499-502.

13 Déjerine J, Déjerine-Klumpke H. Anatomie des centres nerveux, vol 2. Paris: J Rueff, 1901, 1-206, 543-50, 617-18.

14 Iwata M. Unilateral palatal paralysis caused by lesion in the corticobulbar tract. Arch Neurol 1984;41:782-4.

15 Rousseaux M, Lesoin F, Quint S. Unilateral pseudobulbar syndrome with limited capsulothalamic infarction. Eur Neurol 1987;27:227-30.

16 Ertekin C, Turman B, Tarlaci S, et al. Cricopharyngeal sphincter muscle responses to transcranial magnetic stimulation in normal subjects and in patients with dysphagia. Clin Neurophysiol 2001;112:86-94. 